Images in Thorax

\title{
Virtual bronchoscopy and 3D spiral CT reconstructions in the management of kyphosis induced tracheal compression
}

\begin{abstract}
A n 85 year old woman presented to the Accident \& Emergency department with a fractured neck of humerus. She had a history of severe osteoporosis and was being treated with alendronic acid and calcium carbonate. Physical examination revealed marked kyphosis with a 90 degree forward curvature of the thoracic spine. Following treatment of her humeral fracture with a collar and cuff, she developed stridor and then a respiratory arrest
\end{abstract}

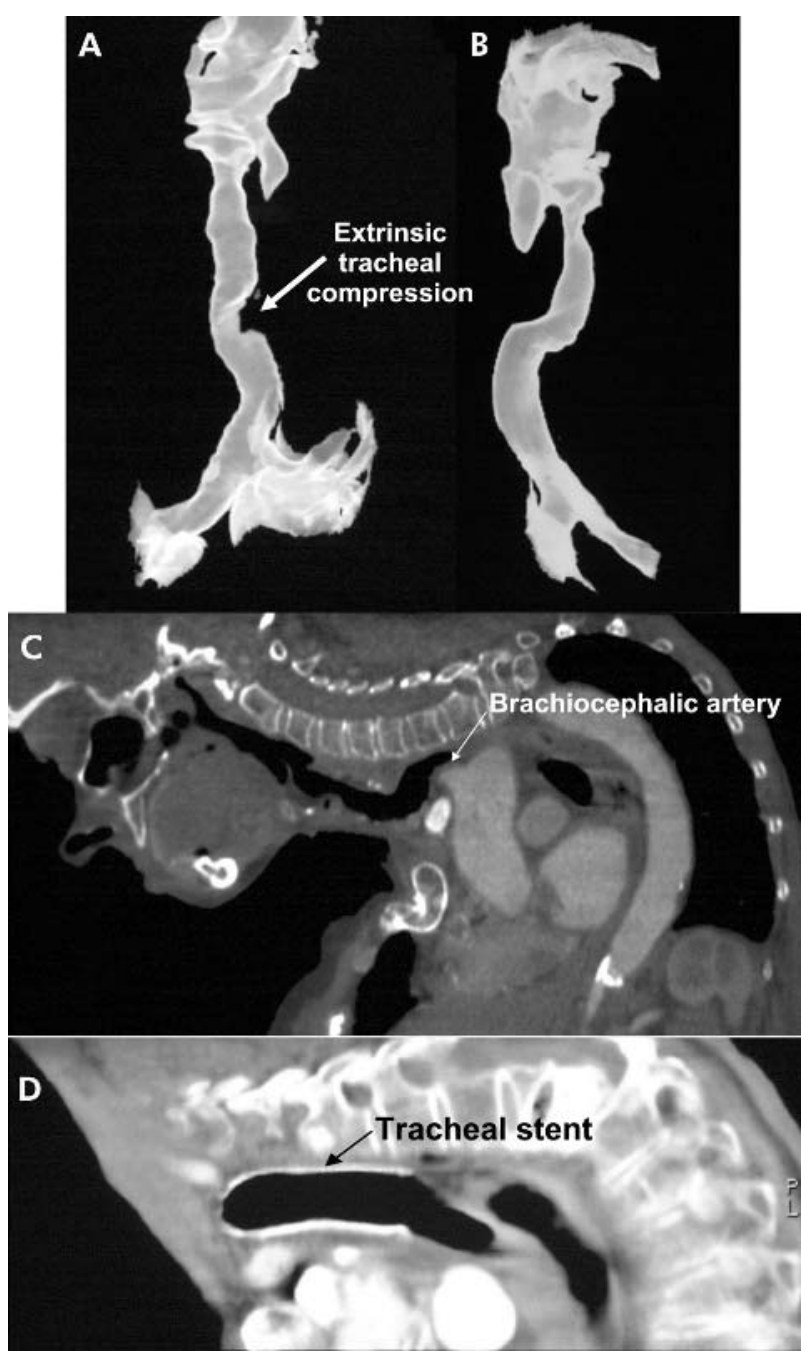

Figure 1 (A) and (B) Spiral three dimensional CT reconstructions showing preoperative distortion of the mid trachea and postoperative restoration of tracheal patency. (C) and (D) Sagittal sections showing compression of the trachea against the brachiocephalic artery anteriorly and postoperative positioning of the metallic stent.

\section{Learning points}

- Virtual bronchoscopy gives a retroverted view of bronchial narrowing.

- Three dimensional spiral CT scanning allows reconstruction of the trachea to show the aetiology of narrowing.

requiring resuscitation and non-invasive positive pressure ventilation. On further questioning the patient described previous episodes of these attacks of stridor. They were not related to any specific triggers and resolved spontaneously after approximately 10 minutes.

Initial investigation with fibreoptic bronchoscopy revealed extrinsic compression of the mid trachea, with the lumen narrowed to less than $5 \mathrm{~mm}$. Pulsation in the anterior wall of the trachea was also noted. In view of this, a spiral CT scan was performed with the patient in a lateral decubitus position as severe kyphosis prevented her lying supine. With the aid of three dimensional reconstructions, forward deviation of the trachea by a markedly kyphotic thoracic spine was found, compressing the airway against the brachiocephalic artery anteriorly (fig 1A and C). Using virtual bronchoscopy to give a "retroverted" view at this non-traversable lesion, a normal tracheobronchial tree was observed distal to the compression. There were no other lesions in the mediastinum or lungs. In view of this, a $6 \mathrm{~cm}(18 \mathrm{~mm})$ diameter Ultraflex non-covered metal tracheobronchial stent (Boston Scientific, USA) was inserted via a rigid bronchoscope under fluoroscopic guidance. A postoperative CT scan showed restoration of airway patency and positioning of the tracheal stent (fig $1 \mathrm{~B}$ and D). On subsequent follow up at 6 weeks the patient was asymptomatic and has not suffered any further attacks of stridor or respiratory distress.

In conclusion, we have reported an unusual case of extrinsic tracheal compression secondary to severe kyphoscoliosis. The use of three dimensional spiral CT reconstructions and virtual bronchoscopy facilitated the insertion of an expandable metal stent, providing excellent symptomatic relief.

N Moorjani, G Conn, J S Rahamim Department of Thoracic Surgery, Derriford Hospital, Plymouth PL6 8DH, UK

N J Ring

Department of Radiology, Derriford Hospital, Plymouth PL6 8DH, UK

Correspondence to: Mr N Moorjani, Department of Thoracic Surgery, Derriford Hospital, Plymouth PL6 8DH, UK; narain.moorjani@doctors.org.uk 